\title{
BERNSTEIN FRACTAL APPROXIMATION AND FRACTAL FULL MÜNTZ THEOREMS*
}

\author{
VIJENDER NALLAPU ${ }^{\dagger}$
}

\begin{abstract}
Fractal interpolation functions defined by means of suitable Iterated Function Systems provide a new framework for the approximation of continuous functions defined on a compact real interval. Convergence is one of the desirable properties of a good approximant. The goal of the present paper is to develop fractal approximants, namely Bernstein $\alpha$-fractal functions, which converge to the given continuous function even if the magnitude of the scaling factors does not approach zero. We use Bernstein $\alpha$-fractal functions to construct the sequence of Bernstein Müntz fractal polynomials that converges to either $f \in \mathcal{C}(I)$ or $f \in L^{p}(I), 1 \leq p<\infty$. This gives a fractal analogue of the full Müntz theorems in the aforementioned function spaces. For a given sequence $\left\{f_{n}(x)\right\}_{n=1}^{\infty}$ of continuous functions that converges uniformly to a function $f \in \mathcal{C}(I)$, we develop a double sequence $\left\{\left\{f_{n, l}^{\alpha}(x)\right\}_{l=1}^{\infty}\right\}_{n=1}^{\infty}$ of Bernstein $\alpha$-fractal functions that converges uniformly to $f$. By establishing suitable conditions on the scaling factors, we solve a constrained approximation problem of Bernstein $\alpha$-fractal Müntz polynomials. We also study the convergence of Bernstein fractal Chebyshev series.
\end{abstract}

Key words. Bernstein polynomials, fractal approximation, convergence, full Müntz theorems, Chebyshev series, box dimension.

AMS subject classifications. 41A30, 28A80, 41A17, 41A50.

1. Introduction. Fractal interpolation and approximation methods are more versatile than traditional nonrecursive approximation methods. Over the last three decades, researchers' interest in fractal functions has steadily grown [1, 3, 4, 5, 6, 7, 8, 9, 18, 19, 29, 31]. It seems unsuitable to use traditional approximants to approximate non-smooth functions. In contrast to traditional methods, the fractal approach provides smooth or non-smooth approximants depending on the nature of the function to be approximated. The theory of Iterated Function System (IFS) and the Read-Bajraktarević operator defined on suitable function spaces are the building blocks of fractal approximants. The fractal dimension is an index that quantifies the irregularity/fractality of the fractal approximant.

Given a continuous function $f$ defined on a real compact interval, Barnsley in [1] and Navascués et al. in $[18,19]$ have identified the suitable IFS to construct a continuous fractal function $f^{\alpha}$ that approximates the function $f$. All the existing fractal approximants, such as those studied in $[16,17,18,19,20,21,22,23,24]$, converge to the given continuous function $f$ provided that the magnitude of the corresponding scaling factors approaches zero. In this paper we develop a sequence of Bernstein $\alpha$-fractal functions that converges uniformly to $f$ even when the magnitude of the scaling factors does not approach zero. We use the Bernstein polynomials of $f$ as base functions to build the Bernstein $\alpha$-fractal functions. The convergence of Bernstein $\alpha$-fractal functions to $f$ follows from the convergence of Bernstein polynomials of $f$ to $f$.

Müntz [14] introduced Müntz polynomials in 1914. By including more general sequences of exponents than just those treated by Müntz, Szász proved the original Müntz theorem in [28]. The classical Müntz theorem was only stated for increasing sequences of nonnegative real numbers. A general result that deals with arbitrary sequences of exponents is referred to as the full Müntz theorem. From Müntz's and Szász's theorems it is clear that the origin plays a very important role in these results, and extending them to spaces of functions defined away from the origin was a nontrivial task. Schwartz [26] proved a full Müntz theorem for

* Received October 23, 2017. Accepted January 12, 2019. Published online on February 21, 2019. Recommended by F. Marcellán.

${ }^{\dagger}$ Mathematics Division, Vellore Institute of Technology Chennai, Chennai 600127, India

(vijendernallapu@gmail.com). 
the space $L^{2}[a, b]$ of square-integrable functions. Schwartz also characterized the density of the Müntz space in $\mathcal{C}[a, b]$ for $0 \notin[a, b]$ and conjectured a necessary and sufficient condition for the density of the space of Müntz polynomials in $\mathcal{C}[0,1]$ with general sequences of real numbers. A few years later Siegel [27] generalized Szász's theorem and proved Schwartz's conjecture by using complex variable techniques. Borwein and Erdélyi [2] largely contributed to research on the full Müntz theorem. The full Müntz theorem [2] establishes the condition on the sequence $\left\{\lambda_{j}\right\}_{j=1}^{\infty}$ of real numbers so that the Müntz space $\operatorname{Span}\left\{x^{\lambda_{j}}: j \in \mathbb{N}\right\}$ is dense in $\mathcal{C}[0,1]$, where $\operatorname{Span}\left\{x^{\lambda_{j}}: j \in \mathbb{N}\right\}$ denotes the collection of all finite linear combinations of the continuous functions $x^{\lambda_{j}}, j \in \mathbb{N}$. The full Müntz theorem [2] of $\mathcal{C}[0,1]$ includes Müntz's second theorem [10] of $\mathcal{C}[0,1]$. Similarly, the classical full Müntz theorem of $L^{p}[0,1], 1 \leq p \leq \infty$, includes Müntz's first theorem [10] of $L^{2}[0,1]$.

Navascués and Chand [22] studied the fractal version of Müntz's first and second theorems by assuming that the magnitude of the corresponding scaling factors tends to zero. In this paper we provide the fractal analogues of the classical full Müntz theorems without imposing any condition on the scaling factors.

Müntz polynomials play a vital role in economics [25] and statistics [12]. In this article, for a given function $f \in \mathcal{C}(I)$, we construct a sequence $\left\{f_{n}^{\alpha}(x)\right\}_{n=1}^{\infty}$ of Bernstein $\alpha$-fractal functions that converges uniformly to $f$ with respect to the supremum norm or the $L^{p}$-norm. We use Bernstein $\alpha$-fractal functions to study the density of fractal Müntz polynomials in $L^{p}[0,1], 1 \leq p<\infty$, and $\mathcal{C}[0,1]$. To define Bernstein $\alpha$-fractal Müntz polynomials of $x^{\lambda_{j}} \in L^{p}[0,1]$ and $x^{\lambda_{j}} \notin \mathcal{C}[0,1]$, we use the density of $\mathcal{C}[0,1]$ in $L^{p}[0,1]$. Under suitable hypotheses, we prove that $\bigcup_{n=1}^{\infty} \operatorname{Span}\left\{f_{j, n}^{\alpha}: j \in \mathbb{N}\right\}$ is dense in $\mathcal{C}[0,1]$ and $L^{p}[0,1]$ if $\operatorname{Span}\left\{f_{j}: j \in \mathbb{N}\right\}$ is, where $f_{j, n}^{\alpha}$ is the Bernstein $\alpha$-fractal function of $f_{j}$. For two given continuous Müntz polynomials $\Phi$ and $\Psi$ such that $\Phi>\Psi$, obtaining a fractal approximant of $\Phi$ that is greater than $\Psi$ constitutes the constrained approximation problem. We address this problem in the present paper. We also study the convergence of a fractal version of Chebyshev series. Overall, this article can also be viewed as an attempt to blend fractal functions and Bernstein polynomials in order to establish a Bernstein-type fractal approximation.

2. Rudiments of fractal approximation theory. To make this paper self-contained, we shall briefly recall here the development of fractal interpolation and approximation.

2.1. Basics of fractal interpolation. Let $(X, d)$ be a complete metric space and $H(X)$ be the class of all non-empty compact subsets of $X$. The set $H(X)$ is a complete metric space with respect to the Hausdorff metric $h$ [1]. Let there be $N-1$ contraction maps $w_{i}: X \rightarrow X$, $i \in \mathbb{N}_{N-1}=\{1,2, \ldots, N-1\}$. Then $\mathcal{I} \equiv\left\{X ; w_{i}, i \in \mathbb{N}_{N-1}\right\}$ is called an Iterated Function System (IFS). If the map $w_{i}$ is a contraction with contraction factor $s_{i}$, for all $i \in \mathbb{N}_{N-1}$, then they induce a set-valued function $W: H(X) \rightarrow H(X), W(E)=\bigcup_{i=1}^{N-1} w_{i}(E)$ which is a contraction on $H(X)$ with a contraction factor $s=\max \left\{s_{i}: i \in \mathbb{N}_{N-1}\right\}$. By the Banach fixed point theorem there exists a unique set $A \in H(X)$ which is invariant with respect to $W$, i.e., $A=W(A)$. The set $A \in H(X)$ is called the attractor of the IFS $\mathcal{I}$. If $\mathcal{I}$ is suitably defined, then $A$ represents the graph of a Fractal Interpolation Function (FIF) based on the following Proposition 2.1. Before stating the result, we recall some notation.

Let $x_{1}<x_{2}<\cdots<x_{N-1}<x_{N}$ for $(N>2)$ be a partition of the closed interval $I=\left[x_{1}, x_{N}\right]$, and let $y_{1}, y_{2}, \ldots, y_{N}$ be a collection of real numbers. Let $L_{i}$ for all $i \in \mathbb{N}_{N-1}$ be homeomorphisms from $I$ to $I_{i}=\left[x_{i}, x_{i+1}\right]$ such that

$$
L_{i}\left(x_{1}\right)=x_{i} \quad \text { and } \quad L_{i}\left(x_{N}\right)=x_{i+1} .
$$

Let then $K$ be a large enough compact subset of $\mathbb{R}$. Let $F_{i}$ be a function from $I \times K$ to $K$, which is continuous in the $x$-direction and contractive in the $y$-direction with a contractive 
factor $\left|\alpha_{i}\right| \leq \kappa<1$ such that

$$
F_{i}\left(x_{1}, y_{1}\right)=y_{i}, \quad F_{i}\left(x_{N}, y_{N}\right)=y_{i+1}, \quad \text { for } i \in \mathbb{N}_{N-1} .
$$

We define the mappings $w_{i}: I \times K \rightarrow I_{i} \times K$ for $i \in \mathbb{N}_{N-1}$ as $w_{i}(x, y)=\left(L_{i}(x), F_{i}(x, y)\right)$, for $(x, y) \in I \times K$.

PROPOSITION 2.1 ([1]). For the given IFS $\left\{I \times K ; w_{i}, i \in \mathbb{N}_{N-1}\right\}$, there exists a unique compact set $A \subset \mathbb{R}^{2}$ such that $W(A)=A$. In addition, there exists a unique continuous function $f$ which satisfies $f\left(x_{i}\right)=y_{i}$ for all $i \in \mathbb{N}_{N}=\{1,2, \ldots, N\}$, and $A$ is the graph of $f$ on $I$. The above function $f$ is called a FIF associated with the IFS $\left\{I \times K ; w_{i}, \in \mathbb{N}_{N-1}\right\}$.

We now recall the functional equation of $f$. Let

$$
\mathcal{G}=\left\{g: I \rightarrow \mathbb{R} \text { continuous } \mid g\left(x_{1}\right)=y_{1}, g\left(x_{N}\right)=y_{N}\right\} .
$$

We define a metric $\rho$ on $\mathcal{G}$ as $\rho(h, g)=\max \{|h(x)-g(x)|: x \in I\}$ for $h, g \in \mathcal{G}$. Then $(\mathcal{G}, \rho)$ is a complete metric space. We define the Read-Bajraktarević operator $T$ on $(\mathcal{G}, \rho)$ by

$$
\operatorname{Tg}(x)=F_{i}\left(L_{i}^{-1}(x), g \circ L_{i}^{-1}(x)\right), \quad \text { for } x \in I_{i} .
$$

Using the properties of $L_{i}$ and (2.1) and (2.2), it can be verified that $T g$ is continuous on the intervals $I_{i}$, for $i \in \mathbb{N}_{N-1}$, at the points $x_{2}, \ldots, x_{N-1}$. Also it is easy to see that $T$ is a contraction map on the complete metric space $(\mathcal{G}, \rho)$, i.e., $\rho(T g, T h) \leq|\alpha|_{\infty} \rho(g, h)$, where $|\alpha|_{\infty}=\max \left\{\left|\alpha_{i}\right|: i \in \mathbb{N}_{N-1}\right\}<1$. Therefore, by the Banach fixed point theorem, $T$ possesses a unique fixed point $f^{*}$ on $\mathcal{G}$, i.e., $\left(T f^{*}\right)(x)=f^{*}(x)$ for all $x \in I$. From (2.3) it follows that the FIF $f^{*}$ satisfies the functional equation $f^{*}(x)=F_{i}\left(L_{i}^{-1}(x), f^{*} \circ L_{i}^{-1}(x)\right)$, for $x \in I_{i}$ and for $i \in \mathbb{N}_{N-1}$. In the present constructions of FIFs, $L_{i}(x)$ and $F_{i}(x, y)$ are defined as

$$
w_{i}(x, y)=\left[\begin{array}{c}
L_{i}(x) \\
F_{i}(x, y)
\end{array}\right]=\left[\begin{array}{c}
a_{i} x+b_{i} \\
\alpha_{i} y+q_{i}(x)
\end{array}\right]
$$

where $a_{i}=\frac{x_{i+1}-x_{i}}{x_{N}-x_{1}}, b_{i}=\frac{x_{N} x_{i}-x_{1} x_{i+1}}{x_{N}-x_{1}},\left|\alpha_{i}\right| \leq \kappa<1$, and the $q_{i}$, for $i \in \mathbb{N}_{N-1}$, are suitable continuous real-valued functions defined on $I$ and such that (2.2) is satisfied.

2.2. $\boldsymbol{\alpha}$-fractal function. Barnsley [1] observed that the concept of FIFs can be used to define a class of fractal functions associated with a given real-valued continuous function $f$ defined on $I=\left[x_{1}, x_{N}\right]$. Later, Navascués $[19,21]$ studied fractal approximations in detail. For a given $f \in \mathcal{C}(I)$, consider a partition $\Delta=\left\{x_{1}, x_{2}, \ldots, x_{N}\right\}$ of $I$ satisfying $x_{1}<x_{2}<\cdots<x_{N}$, a continuous function $b: I \rightarrow \mathbb{R}$ that fulfills $b\left(x_{1}\right)=f\left(x_{1}\right)$, $b\left(x_{N}\right)=f\left(x_{N}\right)$, and $b \neq f$, and $(N-1)$ real numbers $\alpha_{i}$, for $i \in \mathbb{N}_{N-1}$, such that $\left|\alpha_{i}\right|<1$. Define an IFS through the maps

$$
L_{i}(x)=a_{i} x+b_{i}, \quad F_{i}(x, y)=\alpha_{i} y+f\left(L_{i}(x)\right)-\alpha_{i} b(x), \quad \text { for } i \in \mathbb{N}_{N-1} .
$$

The corresponding FIF, denoted by $f_{\Delta, b}^{\alpha}=f^{\alpha}$, is referred to as $\alpha$-fractal function or fractal approximation for $f$ with respect to a scaling vector $\alpha=\left(\alpha_{1}, \alpha_{2}, \ldots, \alpha_{N-1}\right)$, a base function $b$, and a partition $\Delta$. Here the set of data points is $\left\{\left(x_{i}, f\left(x_{i}\right)\right): i \in \mathbb{N}_{N}\right\}$. The function $f^{\alpha}$ is the fixed point of the Read-Bajraktarević (RB) operator $T^{\alpha}: \mathcal{C}_{f}(I) \rightarrow \mathcal{C}_{f}(I)$ defined by

$$
\left(T^{\alpha} g\right) x=\alpha_{i} g\left(L_{i}^{-1}(x)\right)+f(x)-\alpha_{i} b\left(L_{i}^{-1}(x)\right), \quad x \in I_{i}, i \in \mathbb{N}_{N-1},
$$

where $\mathcal{C}_{f}(I)=\left\{g \in \mathcal{C}(I): g\left(x_{1}\right)=f\left(x_{1}\right), g\left(x_{N}\right)=f\left(x_{N}\right)\right\}$. Consequently, the $f^{\alpha}$-fractal function corresponding to $f$ satisfies the equation

$$
f^{\alpha}(x)=\alpha_{i} f^{\alpha}\left(L_{i}^{-1}(x)\right)+f(x)-\alpha_{i} b\left(L_{i}^{-1}(x)\right), \quad x \in I_{i}, i \in \mathbb{N}_{N-1} .
$$


The fractal dimension (box dimension or Hausdorff dimension) of the function $f^{\alpha}$ depends on the choice of the scaling vector $\alpha$. Nasim Akhtar et al. [15] derived the box dimension of the graph of $\alpha$-fractal functions by assuming suitable conditions on the original function $f$ and the base function $b$. The following result provides the details.

Proposition 2.2. Let $f \in \mathcal{C}(I)$ and $b: I \rightarrow \mathbb{R}$ be Lipschitz functions with $b\left(x_{1}\right)=f\left(x_{1}\right)$, $b\left(x_{N}\right)=f\left(x_{N}\right)$. Let $\Delta=\left\{x_{1}, x_{2}, \ldots, x_{N}\right\}$ be a partition of I with $x_{1}<x_{2}<\cdots<x_{N}$, and let $\alpha=\left(\alpha_{1}, \alpha_{2}, \ldots, \alpha_{N-1}\right)$. If the data points $\left(x_{i}, f\left(x_{i}\right)\right)$, for $i \in \mathbb{N}_{N}$, are not collinear, then the graph $G$ of the $\alpha$-fractal function $f^{\alpha}$ has box dimension

$$
\operatorname{dim}_{B}^{G}= \begin{cases}D & \text { if } \sum_{i=1}^{N-1}\left|\alpha_{i}\right|>1 \\ 1 & \text { otherwise }\end{cases}
$$

where $D$ is the solution of $\sum_{i=1}^{N-1}\left|\alpha_{i}\right| a_{i}^{D-1}=1$.

The following proposition provides sufficient conditions for the set of points of nondifferentiability of $f^{\alpha}$ to be dense in $I$. Its proof follows from [18, Lemma 5.1] and [18, Theorem 5.2] and is therefore omitted.

Proposition 2.3. Let $f, b \in \mathcal{C}^{1}(I)$. Let $\Delta=\left\{x_{1}, x_{2}, \ldots, x_{N}\right\}$ be a partition of $I=\left[x_{1}, x_{N}\right]$ satisfying $x_{1}<x_{2}<\cdots<x_{N}$, and let $\alpha=\left(\alpha_{1}, \alpha_{2}, \ldots, \alpha_{N-1}\right)$. If $f^{\prime}(x)$ and $b^{\prime}(x)$ respectively do not agree with $f\left(x_{N}\right)-f\left(x_{1}\right)$ and $N\left(b\left(x_{N}\right)-b\left(x_{1}\right)\right)$ in a nonempty open subinterval of $I$, and $\sum_{i=1}^{N-1}\left|\alpha_{i}\right|>1$, then the set of points of non-differentiability of $f^{\alpha}$ is dense in $I$.

3. Bernstein $\boldsymbol{\alpha}$-fractal approximation. In this section, for $f \in \mathcal{C}(I)$, we develop a sequence of Bernstein $\alpha$-fractal functions that converges uniformly to $f$ for every scaling vector $\alpha$.

From (2.4), the following bound for the uniform error in the process of $\alpha$-fractal approximation can be easily deduced:

$$
\left\|f^{\alpha}-f\right\|_{\infty} \leq \frac{|\alpha|_{\infty}}{1-|\alpha|_{\infty}}\|f-b\|_{\infty}
$$

From (3.1) it follows that for a fixed base function $b$ and a partition $\Delta$, the $\alpha$-fractal function $f^{\alpha}$ converges uniformly to $f \in \mathcal{C}(I)$ if $|\alpha|_{\infty} \rightarrow 0$. To obtain the convergence of $f^{\alpha}$ towards $f$ for every scaling vector $\alpha$, we choose the base function $b$ as the Bernstein polynomial $B_{n}(f, x)$ of $f$, i.e., for all $x \in I, n \in \mathbb{N}$,

$$
b=B_{n}(f, x)=\frac{1}{\left(x_{N}-x_{1}\right)^{n}} \sum_{k=0}^{n}\left(\begin{array}{l}
n \\
k
\end{array}\right)\left(x-x_{1}\right)^{k}\left(x_{N}-x\right)^{n-k} f\left(x_{1}+\frac{k\left(x_{N}-x_{1}\right)}{n}\right) .
$$

It is easy to verify that $B_{n}\left(f, x_{1}\right)=f\left(x_{1}\right), B_{n}\left(f, x_{N}\right)=f\left(x_{N}\right)$ for all $n \in \mathbb{N}$. Then, for every $n \in \mathbb{N}$, the corresponding $\alpha$-fractal function $f^{\alpha}=f_{n}^{\alpha}$ is called Bernstein $\alpha$-fractal function of order $n$ of $f \in \mathcal{C}(I)$, and

$$
f_{n}^{\alpha}(x)=\alpha_{i} f_{n}^{\alpha}\left(L_{i}^{-1}(x)\right)+f(x)-\alpha_{i} B_{n}\left(f, L_{i}^{-1}(x)\right), \quad x \in I_{i}, i \in \mathbb{N}_{N-1}
$$

From the construction of fractal functions (see the previous section), it can be verified that for every $n \in \mathbb{N}$, the Bernstein $\alpha$-fractal function $f_{n}^{\alpha}$ of $f \in \mathcal{C}(I)$ is obtained via the IFS defined by

$$
\mathcal{I}_{n}=\left\{I \times K,\left(L_{i}(x), F_{n, i}(x, y)\right), i \in \mathbb{N}_{N-1}\right\},
$$


where $F_{n, i}(x, y)=\alpha_{i} y+f\left(L_{i}(x)\right)-\alpha_{i} B_{n}(f, x)$. From (3.2) it follows that for any given $f \in \mathcal{C}(I)$ there exists a sequence $\left\{f_{n}^{\alpha}(x)\right\}_{n=1}^{\infty}$ of Bernstein $\alpha$-fractal functions. The following theorem addresses the convergence of the sequence $\left\{f_{n}^{\alpha}(x)\right\}_{n=1}^{\infty}$ towards $f \in \mathcal{C}(I)$.

THEOREM 3.1. Let $\mathcal{C}(I)$ be endowed with the uniform norm $\|\cdot\|_{\infty}$. Let the partition $\Delta=\left\{x_{1}, x_{2}, \ldots, x_{N}\right\}$ of $I=\left[x_{1}, x_{N}\right]$ satisfy $x_{1}<x_{2}<\cdots<x_{N}$, and let $\alpha=\left(\alpha_{1}, \alpha_{2}, \ldots, \alpha_{N-1}\right) \in(-1,1)^{N-1}$. Then, for every scaling vector $\alpha$, the sequence $\left\{\mathcal{I}_{n}\right\}_{n=1}^{\infty}$ of IFSs determines a sequence $\left\{f_{n}^{\alpha}(x)\right\}_{n=1}^{\infty}$ of Bernstein $\alpha$-fractal functions that converges uniformly to $f$ on I with respect to the uniform norm.

Proof. From (3.2), it follows

$$
\left\|f_{n}^{\alpha}-f\right\|_{\infty} \leq|\alpha|_{\infty}\left\|f_{n}^{\alpha}-B_{n}(f, \cdot)\right\|_{\infty}, \leq|\alpha|_{\infty}\left(\left\|f_{n}^{\alpha}-f\right\|_{\infty}+\mid f-B_{n}(f, \cdot) \|_{\infty}\right) .
$$

Hence we obtain

$$
\left\|f_{n}^{\alpha}-f\right\|_{\infty} \leq \frac{|\alpha|_{\infty}}{1-|\alpha|_{\infty}}\left\|f-B_{n}(f, \cdot)\right\|_{\infty}
$$

From classical approximation theory [11], we obtain that $\left\|f-B_{n}(f, \cdot)\right\|_{\infty} \leq \frac{3}{2} \omega_{f}\left(n^{-1 / 2}\right)$. Using this inequality in (3.3), we get

$$
\left\|f_{n}^{\alpha}-f\right\|_{\infty} \leq \frac{3|\alpha|_{\infty}}{2\left(1-|\alpha|_{\infty}\right)} \omega_{f}\left(n^{-1 / 2}\right)
$$

Since $f$ is uniformly continuous on $I$, the modulus of continuity $\omega_{f}\left(n^{-1 / 2}\right) \rightarrow 0$ as $n \rightarrow \infty$. Hence, from (3.4) we can see that the sequence $\left\{f_{n}^{\alpha}(x)\right\}_{n=1}^{\infty}$ of Bernstein $\alpha$-fractal functions converges uniformly to $f$.

The next theorem discusses sufficient conditions which ensure that the box dimension of each Bernstein $\alpha$-fractal function in the sequence $\left\{f_{n}^{\alpha}(x)\right\}_{n=1}^{\infty}$ is greater than one.

THEOREM 3.2. Let $f \in \mathcal{C}(I)$ be a Lipschitz function. Let $\Delta=\left\{x_{1}, x_{2}, \ldots, x_{N}\right\}$ be a partition of $I=\left[x_{1}, x_{N}\right]$ satisfying $x_{1}<x_{2}<\cdots<x_{N}$, and let $\alpha=\left(\alpha_{1}, \alpha_{2}, \ldots, \alpha_{N-1}\right) \in$ $(-1,1)^{N-1}$. If $\sum_{i=1}^{N-1}\left|\alpha_{i}\right|>1$ and the data points $\left(x_{i}, f\left(x_{i}\right)\right)$, for $i \in \mathbb{N}_{N}$, are not collinear, then there exists a sequence $\left\{f_{n}^{\alpha}(x)\right\}_{n=1}^{\infty}$ of Bernstein $\alpha$-fractal functions that converges uniformly to $f$ on I and whose box dimension is greater than one.

Proof. For every $n \in \mathbb{N}$, let $B_{n}(f, x)$ be the Bernstein polynomial of $f$. Since $B_{n}(f, x)$ is continuously differentiable, it is Lipschitz. From Proposition 2.2 it then follows that for every $n \in \mathbb{N}$, the box dimension $\operatorname{dim}_{B}^{G_{n}}$ of the graph $G_{n}$ of the Bernstein $\alpha$-fractal function $f_{n}^{\alpha}$ is greater than one and is the solution to

$$
\sum_{i=1}^{N-1}\left|\alpha_{i}\right| a_{i}^{\operatorname{dim}_{B}^{G n}-1}=1 .
$$

The result then follows from Theorem 3.1.

The proof of the following theorem follows from Proposition 2.3 and Theorem 3.1.

THEOREM 3.3. Let $f \in \mathcal{C}^{1}(I)$. Let $\Delta=\left\{x_{1}, x_{2}, \ldots, x_{N}\right\}$ be a partition of $I=\left[x_{1}, x_{N}\right]$ satisfying $x_{1}<x_{2}<\cdots<x_{N}$, and let $\alpha=\left(\alpha_{1}, \alpha_{2}, \ldots, \alpha_{N-1}\right)$. Suppose that $f^{\prime}(x)$ and $B_{n}^{\prime}(f, x)$ do not respectively agree with $f\left(x_{N}\right)-f\left(x_{1}\right)$ and $N\left(b\left(x_{N}\right)-b\left(x_{1}\right)\right)$ in a nonempty open subinterval of I. If all Bernstein $\alpha$-fractal functions in the sequence $\left\{f_{n}^{\alpha}(x)\right\}_{n=1}^{\infty}$ are obtained with the same choice of scaling factors $\alpha_{i}$, for $i \in \mathbb{N}_{N-1}$, such that $\sum_{i=1}^{N-1}\left|\alpha_{i}\right|>1$, 
then all the Bernstein $\alpha$-fractal functions are non-differentiable in a dense subset of $I$, and the sequence $\left\{f_{n}^{\alpha}(x)\right\}_{n=1}^{\infty}$ converges uniformly to $f$.

REMARK 3.4. For a prescribed continuous function $f$, the proposed Bernstein fractal approximation provides a way of constructing a sequence of $\alpha$-fractal functions that converges to $f$ and has a specified box dimension.

REMARK 3.5. In the construction of fractal interpolants and approximants, the selection of the "base function" is an important task. For instance, using a single base function, Barnsley [1] and Navascués and Sebastián [24], respectively, constructed $\mathcal{C}^{0}$-FIFs and $\mathcal{C}^{r}$-FIFs (polynomial FIFs). By using a finite sequence of base functions, Viswanathan and Chand [29] developed shape-preserving smooth rational FIFs with shape parameters. From [29] and the results in the present paper, one can show that a finite sequence of base functions is suitable for the construction of shape-preserving fractal splines, whereas the sequence of Bernstein polynomials is to be preferred for the construction of $\alpha$-fractal functions that converge to the original function $f$ for any choice of the scaling factors.

THEOREM 3.6. Let $\left\{f_{n}(x)\right\}_{n=1}^{\infty}$ be a sequence of continuous functions on I that converges uniformly to a function $f$. Let $\Delta=\left\{x_{1}, x_{2}, \ldots, x_{N}\right\}$ be a partition of I satisfying $x_{1}<x_{2}<\cdots<x_{N}$, and let $\alpha=\left(\alpha_{1}, \alpha_{2}, \ldots, \alpha_{N-1}\right) \in(-1,1)^{N-1}$. Suppose that $L_{i}: I \rightarrow I_{i}, i \in \mathbb{N}_{N-1}$ are affine maps $L_{i}(x)=a_{i} x+b_{i}$ satisfying $L_{i}\left(x_{1}\right)=x_{i}$ and $L_{i}\left(x_{N}\right)=x_{i+1}$, and let $F_{n, l, i}^{\dagger}(x, y)=\alpha_{i} y+f_{n}\left(L_{i}(x)\right)-\alpha_{i} B_{l}\left(f_{n}, x\right)$, for $i \in \mathbb{N}_{N-1}$ and for all $n, l \in \mathbb{N}$. For every $n, l \in \mathbb{N}$, let $f_{n, l}^{\alpha}$ be $\alpha$-fractal function determined by the IFS $\mathcal{I}_{n, l}^{\dagger}=\left\{I \times K,\left(L_{i}(x), F_{n, l, i}^{\dagger}(x, y)\right), i \in \mathbb{N}_{N-1}\right\}$. Then, for every scaling vector $\alpha$, the double sequence $\left\{\left\{\mathcal{I}_{n, l}^{\dagger}\right\}_{l=1}^{\infty}\right\}_{n=1}^{\infty}$ of IFSs determine a double sequence $\left\{\left\{f_{n, l}^{\alpha}(x)\right\}_{l=1}^{\infty}\right\}_{n=1}^{\infty}$ of Bernstein $\alpha$-fractal functions that converges uniformly to $f$.

Proof. If $\alpha=(0,0, \ldots, 0)$, then it follows that $f_{n, l}^{\alpha}=f_{n}$, for all $n \in \mathbb{N}$, and thus the sequence $\left\{f_{n}\right\}_{n=1}^{\infty}$ converges to $f$. Consider now a non-zero scaling vector. Since $\left\{f_{n}(x)\right\}_{n=1}^{\infty}$ is a sequence of continuous functions on $I$ converging uniformly to a function $f$, for a given $\epsilon$ there exists $N_{1} \in \mathbb{N}$ such that

$$
\left\|f_{n}-f\right\|_{\infty}<\frac{\epsilon}{2} \quad \forall n \geq N_{1} .
$$

From standard results in approximation theory, we can see that for each $n \in \mathbb{N}$, there exists a sequence $\left\{B_{l}\left(f_{n}, x\right)\right\}_{l=1}^{\infty}$ of Bernstein polynomials that converges uniformly to $f_{n}$. Therefore, for a given $\epsilon>0$ there exists $N_{2} \in \mathbb{N}$ such that

$$
\left\|B_{l}\left(f_{n}, \cdot\right)-f_{n}\right\|_{\infty}<\frac{\epsilon\left(1-|\alpha|_{\infty}\right)}{2|\alpha|_{\infty}} \quad \forall l \geq N_{2}
$$

Since $f_{n, l}^{\alpha}$, for $n, l \in \mathbb{N}$, is the $\alpha$-fractal function determined by the IFS $\mathcal{I}_{n, l}^{\dagger}$, it satisfies the following functional equation:

$$
f_{n, l}^{\alpha}(x)=\alpha_{i} f_{n, l}^{\alpha}\left(L_{i}^{-1}(x)\right)+f_{n}(x)-\alpha_{i} B_{l}\left(f_{n}, L_{i}^{-1}(x)\right), \quad x \in I_{i}, i \in \mathbb{N}_{N-1} .
$$

Hence, we get the following inequality:

$$
\left\|f_{n, l}^{\alpha}-f_{n}\right\|_{\infty} \leq \frac{|\alpha|_{\infty}}{1-|\alpha|_{\infty}}\left\|f_{n}-B_{l}\left(f_{n}, \cdot\right)\right\|_{\infty}
$$

Using (3.6) in (3.7), we obtain

$$
\left\|f_{n, l}^{\alpha}-f_{n}\right\|_{\infty}<\frac{\epsilon}{2} \quad \forall l \geq N_{2}
$$


and from (3.5), (3.8) and the triangular inequality it follows that

$$
\left\|f_{n, l}^{\alpha}-f\right\|_{\infty} \leq\left\|f_{n, l}^{\alpha}-f_{n}\right\|_{\infty}+\left\|f_{n}-f\right\|_{\infty}
$$

For a given $\epsilon>0$, we then get

$$
\left\|f_{n, l}^{\alpha}-f\right\|_{\infty}<\epsilon \quad \forall l, n \geq N=\max \left\{N_{1}, N_{2}\right\}
$$

and thus the conclusion $\lim _{n, l \rightarrow \infty} f_{n, l}^{\alpha}=f$.

REMARK 3.7. From Theorem 3.6 it follows that if there exists a sequence of continuous functions that converges to $f \in \mathcal{C}(I)$, then by taking different values for the scaling factors we can construct an infinite number of sequences of Bernstein $\alpha$-fractal functions that converge to $f \in \mathcal{C}(I)$.

THEOREM 3.8. Let $\mathcal{C}(I)$ be endowed with the uniform norm $\|\cdot\|_{\infty}$. For every $n \in \mathbb{N}$, the $\alpha$-operator $\mathcal{F}_{n}^{\alpha}: \mathcal{C}(I) \rightarrow \mathcal{C}(I)$ defined by $\mathcal{F}_{n}^{\alpha}(f)=f_{n}^{\alpha}$ is linear and bounded.

Proof. The linearity of $\mathcal{F}_{n}^{\alpha}$ follows from [16, 29]. From (3.3), we get

$$
\left\|\mathcal{F}_{n}^{\alpha}(f)\right\|_{\infty}=\left\|f_{n}^{\alpha}\right\|_{\infty} \leq\|f\|_{\infty}+\frac{|\alpha|_{\infty}}{1-|\alpha|_{\infty}}\left\|I_{d}-B_{n}(\cdot, \cdot)\right\|_{\infty^{*}}\|f\|_{\infty},
$$

where $I_{d}$ is the identity operator and $\|\cdot\|_{\infty^{*}}$ is the operator norm induced by $\|\cdot\|_{\infty}$. From classical approximation theory, it follows that $\left\|I_{d}-B_{n}(\cdot, \cdot)\right\|_{\infty^{*}} \rightarrow 0$ as $n \rightarrow \infty$, hence $\mathcal{F}_{n}^{\alpha}$ is bounded.

3.1. Examples. In this section, we provide numerical examples to corroborate our findings. For this purpose, let $f(x)=\sin (\pi x)+\cos (\pi x)$ for $x \in[0,1]$. The graph of the function $f(x)=\sin (\pi x)+\cos (\pi x), x \in[0,1]$, is given in Figure 3.1(a). The Bernstein $\alpha$-fractal functions in Figures 3.1(b)-(f) are generated with respect to the partition $\Delta=\{0,0.1111,0.2222,0.3333,0.4444,0.5556,0.6667,0.7778,0.8889,1\}$. The Bernstein $\alpha$-fractal functions $f_{3}^{\alpha}, f_{7}^{\alpha}$, and $f_{26}^{\alpha}$ in Figures 3.1(b)-(d) are generated at the third iteration with the choice of the scaling factors $\alpha_{i}=0.8$, for $i \in \mathbb{N}_{9}$. Since $f(x)=\sin (\pi x)+\cos (\pi x)$ and the Bernstein polynomial $B_{n}(f, x)$ are Lipschitz, the fractal dimension (box dimension) of the Bernstein $\alpha$-fractal functions $f_{3}^{\alpha}, f_{7}^{\alpha}$, and $f_{26}^{\alpha}$ is computed using Proposition 2.2 and equals 1.8984 in all three cases. From Theorem 3.1 it follows that the Bernstein $\alpha$-fractal function $f_{26}^{\alpha}$ provides a better approximation for $\sin (\pi x)+\cos (\pi x), x \in[0,1]$, than those obtained by $f_{3}^{\alpha}$ and $f_{7}^{\alpha}$. By observing Figures 3.1(b)-(d), one may wonder why the fractal functions $f_{3}^{\alpha}, f_{7}^{\alpha}$, and $f_{26}^{\alpha}$ do not display the same sort of irregularity despite having the same fractal dimension. This is due to the following reason: the $\alpha$-fractal functions $f_{3}^{\alpha}$ and $f_{7}^{\alpha}$ exhibit irregularity at all scales whereas the $\alpha$-fractal function $f_{26}^{\alpha}$ exhibits irregularity at small scales only. Small scales of irregularity of the $\alpha$-fractal function $f_{26}^{\alpha}$ can be observed from Figure 3.1(e), where we display a zoom-in of a part of $f_{26}^{\alpha}$. From the discussion above and Theorem 3.3, we conclude that even if the scaling factors $\alpha_{i}$ for $i \in \mathbb{N}_{N-1}$ are chosen such that the condition $\sum_{i=1}^{N-1}\left|\alpha_{i}\right|>1$ is satisfied, for large $n \in \mathbb{N}$, the Bernstein $\alpha$-fractal function $f_{n}^{\alpha}$ may not exhibit irregularity on large scales, but they can certainly do so on small scales. If the scaling factors $\alpha_{i}$, for $i \in \mathbb{N}_{N-1}$, are chosen such that the condition $\sum_{i=1}^{N-1}\left|\alpha_{i}\right|>1$ is satisfied, then for sufficiently large $n, f_{n}^{\alpha}$ provides a good non-differentiable approximation to the original function. Values of the uniform norms $\left\|B_{n}(f, \cdot)-f\right\|_{\infty}$ and $\left\|f_{n}^{\alpha}-f\right\|_{\infty}$, for $n=3,7,26,141$, estimated by using 7290 points in $[0,1]$ that are generated at the third iteration, are given in Table 3.1. From Table 3.1 one can observe that for large $n \in \mathbb{N}$, both the Bernstein polynomial and the Bernstein $\alpha$-fractal function $f_{n}^{\alpha}$ provide almost the same approximation for the given function. However, the box dimension makes the difference 


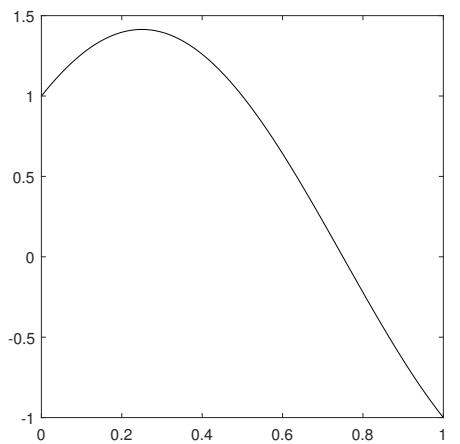

(a): $f(x)=\sin (\pi x)+\cos (\pi x), x \in[0,1]$.

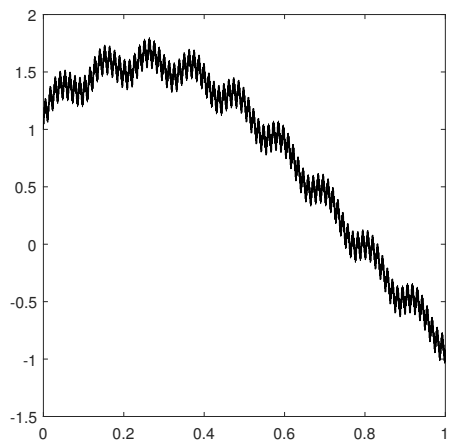

(c): $f_{7}^{\alpha}$ with $\alpha_{i}=0.8, i \in \mathbb{N}_{9}$.

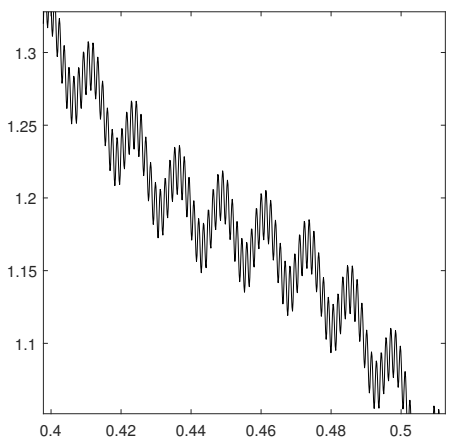

(e): Zoom-in on part of $f_{26}^{\alpha}$.

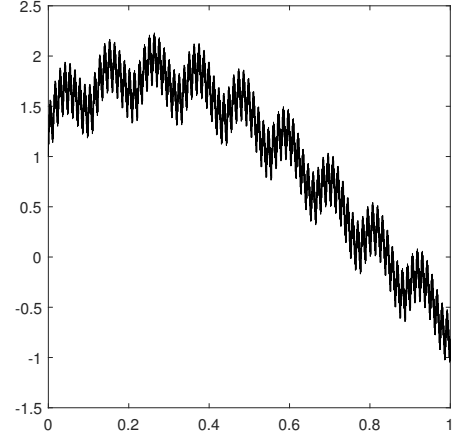

(b): $f_{3}^{\alpha}$ with $\alpha_{i}=0.8, i \in \mathbb{N}_{9}$.

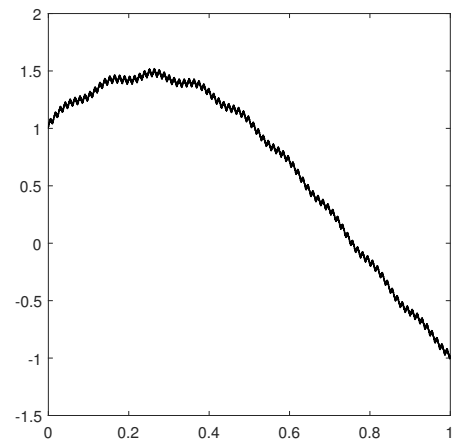

(d): $f_{26}^{\alpha}$ with $\alpha_{i}=0.8, i \in \mathbb{N}_{9}$.

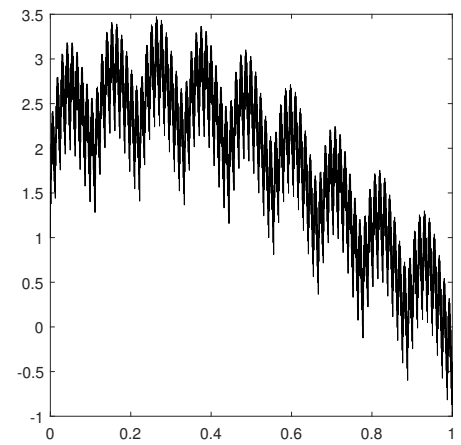

(f): The $\alpha$-fractal function (2.4) with $\alpha_{i}=0.8, i \in \mathbb{N}_{9}$ and the base function $b(x)=1-2 x, x \in[0,1]$.

FIG. 3.1. Bernstein $\alpha$-fractal approximants of $\sin (\pi x)+\cos (\pi x), x \in[0,1]$.

between the Bernstein polynomial and the Bernstein $\alpha$-fractal function. For a given function, if one needs an approximant whose box dimension is greater than one, then our Bernstein $\alpha$-fractal function can be chosen. The $\alpha$-fractal function $f^{\alpha}$ with $\alpha_{i}=0.8$, for $i \in \mathbb{N}_{9}$, and a base function $b(x)=1-2 x, x \in[0,1]$, generated at the third iteration are displayed in Figure 3.1(f). Here, $\left\|f^{\alpha}-f\right\|_{\infty}=4.4720$. 


\section{ETNA}

Kent State University and

Johann Radon Institute (RICAM)

BERNSTEIN FRACTAL APPROXIMATION AND FRACTAL FULL MÜNTZ THEOREMS

TABLE 3.1

Error in Bernstein approximation and Bernstein fractal approximation for $\sin (\pi x)+\cos (\pi x), x \in[0,1]$.

\begin{tabular}{l|l} 
Error in Bernstein approximation & Error in Bernstein $\alpha$-fractal approximation
\end{tabular}

\begin{tabular}{|c|c|}
\hline $\begin{aligned}\left\|B_{3}(f, \cdot)-f\right\|_{\infty} & =2.4142 \\
\left\|B_{7}(f, \cdot)-f\right\|_{\infty} & =2.4142 \\
\left\|B_{26}(f, \cdot)-f\right\|_{\infty} & =2.4142 \\
\mid B_{141}(f, \cdot)-f \|_{\infty} & =2.4142\end{aligned}$ & $\begin{aligned}\left\|f_{3}^{\alpha}-f\right\|_{\infty} & =3.2299 \\
\left\|f_{7}^{\alpha}-f\right\|_{\infty} & =2.7980 \\
\left\|f_{26}^{\alpha}-f\right\|_{\infty} & =2.5200 \\
\left\|f_{141}^{\alpha}-f\right\|_{\infty} & =2.4341\end{aligned}$ \\
\hline
\end{tabular}

4. Bernstein $\alpha$-fractal full Müntz theorems. In this section, we introduce Bernstein $\alpha$-fractal Müntz polynomials and the fractal version of "full Müntz theorems" in the spaces $\mathcal{C}[0,1]$ and $L^{p}[0,1]$, for $1 \leq p<\infty$. For $f \in L^{p}(I)$, let $\|f\|_{L^{p}}=\left(\int_{x_{1}}^{x_{N}}|f(x)|^{p} d x\right)^{\frac{1}{p}}$, for $1 \leq p<\infty$. Next we define the $\alpha$-operator on $L^{p}(I)$. Since $\mathcal{C}(I)$ is dense in $L^{p}(I)$, $1 \leq p<\infty$, for every $f \in L^{p}(I)$ there exists a sequence $\left\{g_{k}(x)\right\}_{k=1}^{\infty}$ in $\mathcal{C}(I)$ that converges to $f$. We can hence define the $\alpha$-operator $\hat{\mathcal{F}}_{n}^{\alpha}: L^{p}(I) \rightarrow L^{p}(I)$ as follows:

$$
\hat{\mathcal{F}}_{n}^{\alpha}(f)= \begin{cases}\mathcal{F}_{n}^{\alpha}(f)=f_{n}^{\alpha} \quad \forall n \in \mathbb{N} & \text { if } f \in \mathcal{C}(I) \cap L^{p}(I), \\ \lim _{k \rightarrow \infty} \mathcal{F}_{n}^{\alpha}\left(g_{k}\right)=\lim _{k \rightarrow \infty}\left(g_{k}\right)_{n}^{\alpha} & \text { if } f \in L^{p}(I) \text { and } f \notin \mathcal{C}(I),\end{cases}
$$

where $\left(g_{k}\right)_{n}^{\alpha}(x)=\alpha_{i}\left(g_{k}\right)_{n}^{\alpha}\left(L^{-1}(x)\right)+g_{k}(x)-\alpha_{i} B_{n}\left(g_{k}, L^{-1}(x)\right), x \in I_{i}, i \in \mathbb{N}_{N-1}$. It can be shown that $\hat{\mathcal{F}}_{n}^{\alpha}$ is linear and bounded using similar arguments to those used to prove Theorem 3.8. Let $\Lambda=\left\{\lambda_{j}\right\}_{j=1}^{\infty}$ be a sequence of distinct real numbers. The collection $\left\{x^{\lambda_{1}}, x^{\lambda_{2}}, \ldots, x^{\lambda_{m}}\right\}$ is called a finite Müntz system. The linear space

$$
\Pi_{m}(\Lambda)=\operatorname{Span}\left\{x^{\lambda_{1}}, x^{\lambda_{2}}, \ldots, x^{\lambda_{m}}\right\}=\left\{\sum_{j=1}^{m} c_{j} x^{\lambda_{j}}: c_{j} \in \mathbb{R}\right\}
$$

is called a finite Müntz space. The set $\Pi(\Lambda)=\bigcup_{m=1}^{\infty} \Pi_{m}(\Lambda)=\operatorname{Span}\left\{x^{\lambda_{1}}, x^{\lambda_{2}}, \ldots\right\}$ is called the infinite Müntz space pertaining to $\Lambda$. Therefore, a Müntz polynomial $p$ in $\Pi(\Lambda)$ takes the form $p(x)=\sum_{j=1}^{k} c_{j} x^{\lambda_{j}}, x \in[0,1], c_{j} \in \mathbb{R}$. Let $\Delta=\left\{x_{1}, x_{2}, \ldots, x_{N}\right\}$ be a partition of $I=[0,1]$ satisfying $0=x_{1}<x_{2}<\cdots<x_{N}=1$, and $\alpha=\left(\alpha_{1}, \alpha_{2}, \ldots, \alpha_{N-1}\right) \in$ $(-1,1)^{N-1}$ be a scaling vector. Based on the partition $\Delta$, the linearity, and the definition of $\hat{\mathcal{F}}_{n}^{\alpha}$, we obtain that

$$
\hat{\mathcal{F}}_{n}^{\alpha}(p(x))=p_{n}^{\alpha}(x)=\sum_{j=1}^{k} c_{j} \hat{\mathcal{F}}_{n}^{\alpha}\left(x^{\lambda_{j}}\right), \quad x \in[0,1], c_{j} \in \mathbb{R}, \forall n \in \mathbb{N},
$$

where

$$
\hat{\mathcal{F}}_{n}^{\alpha}\left(x^{\lambda_{j}}\right)= \begin{cases}\mathcal{F}_{n}^{\alpha}\left(x^{\lambda_{j}}\right)=\left(x^{\lambda_{j}}\right)_{n}^{\alpha} & \text { if } \lambda_{i} \geq 0, \\ \lim _{k \rightarrow \infty} \mathcal{F}_{n}^{\alpha}\left(h_{k}\right)=\lim _{k \rightarrow \infty}\left(h_{k}\right)_{n}^{\alpha} & \text { if }-1 / p<\lambda_{i}<0,\end{cases}
$$

$\left(h_{k}\right)_{n}^{\alpha}(x)=\alpha_{i}\left(h_{k}\right)_{n}^{\alpha}\left(L^{-1}(x)\right)+h_{k}(x)-\alpha_{i} B_{n}\left(h_{k}, L^{-1}(x)\right), x \in I_{i}, i \in \mathbb{N}_{N-1}$, and $\left\{h_{k}\right\}_{k=1}^{\infty}$ is the sequence in $\mathcal{C}[0,1]$ that converges to $x^{\lambda_{j}}$ for $-1 / p<\lambda_{i}<0$. It is worthwhile to mention that $x^{\lambda_{j}} \in L^{p}[0,1]$ if $-1 / p<\lambda_{i}<0$. Since $\left\{x^{\lambda_{j}}, j=1,2, \ldots, m\right\}$ is a linearly independent set, it follows that $\left\{\hat{\mathcal{F}}_{n}^{\alpha}\left(x^{\lambda_{j}}\right), j=1,2, \ldots, m\right\}$ is also a linearly independent set, and hence it forms a basis for the fractal Müntz space

$$
\Pi_{m, n}^{\alpha}(\Lambda)=\operatorname{Span}\left\{\hat{\mathcal{F}}_{n}^{\alpha}\left(x^{\lambda_{1}}\right), \hat{\mathcal{F}}_{n}^{\alpha}\left(x^{\lambda_{2}}\right), \ldots, \hat{\mathcal{F}}_{n}^{\alpha}\left(x^{\lambda_{m}}\right)\right\}=\left\{\sum_{j=1}^{m} c_{j} \hat{\mathcal{F}}_{n}^{\alpha}\left(x^{\lambda_{j}}\right): c_{j} \in \mathbb{R}\right\} .
$$


THEOREM 4.1 (Fractal version of the full Müntz theorem in $\mathcal{C}[0,1]$ ). Let the partition $\Delta=\left\{x_{1}, x_{2}, \ldots, x_{N}\right\}$ of $I=[0,1]$ satisfy $0=x_{1}<x_{2}<\cdots<x_{N}=1$, and let $\alpha=\left(\alpha_{1}, \alpha_{2}, \ldots, \alpha_{N-1}\right) \in(-1,1)^{N-1}$ be a scaling vector. Suppose that $\left\{\lambda_{j}\right\}_{j=1}^{\infty}$ is a sequence of distinct and positive real numbers. Then, for every scaling vector $\alpha$, the set $\bigcup_{n=1}^{\infty} \operatorname{Span}\left\{1,\left(x^{\lambda_{1}}\right)_{n}^{\alpha},\left(x^{\lambda_{2}}\right)_{n}^{\alpha}, \ldots,\right\}$ is dense in $\mathcal{C}[0,1]$ with respect to the supremum norm if $\sum_{j=1}^{\infty} \frac{\lambda_{j}}{\lambda_{j}^{2}+1}=\infty$.

Proof. Let $f \in \mathcal{C}[0,1]$ and $\epsilon>0$ be given. From the classical full Müntz theorem [2] in $\mathcal{C}[0,1]$, we obtain that $\operatorname{Span}\left\{1, x^{\lambda_{1}}, x^{\lambda_{2}}, \ldots,\right\}$ is dense in $\mathcal{C}[0,1]$ with respect to the supremum norm if $\sum_{j=1}^{\infty} \frac{\lambda_{j}}{\lambda_{j}^{2}+1}=\infty$, where $\left\{\lambda_{j}\right\}_{j=1}^{\infty}$ is a sequence of distinct and positive real numbers. Hence, there exists a Müntz polynomial $Q=\sum_{j=1}^{\mu} d_{j} x^{\lambda_{j}}, d_{j} \in \mathbb{R}$, such that

$$
\|f-Q\|_{\infty}<\frac{\epsilon}{2}
$$

From (4.1), we get

$$
\hat{\mathcal{F}}_{n}^{\alpha}(Q)=Q_{n}^{\alpha}=\sum_{j=1}^{\mu} d_{j}\left(x^{\lambda_{j}}\right)_{n}^{\alpha}, \quad \forall n \in \mathbb{N} .
$$

Now from $Q-Q_{n}^{\alpha}=\sum_{j=1}^{\mu} d_{j}\left(x^{\lambda_{j}}-\left(x^{\lambda_{j}}\right)_{n}^{\alpha}\right)$ it follows that

$$
\left\|Q-Q_{n}^{\alpha}\right\|_{\infty} \leq \sum_{j=1}^{\mu}\left|d_{j}\right|\left\|x^{\lambda_{j}}-\left(x^{\lambda_{j}}\right)_{n}^{\alpha}\right\|_{\infty} .
$$

Using Theorem 3.1, one can observe that the sequence $\left\{\left(x^{\lambda_{j}}\right)_{n}^{\alpha}\right\}_{n=1}^{\infty}$ converges uniformly to $x^{\lambda_{j}}$ for each $j=1,2, \ldots, \mu$. Hence, for given $\epsilon>0$, there exists $n_{j} \in \mathbb{N}$ such that

$$
\left\|\left(x^{\lambda_{j}}\right)_{n}^{\alpha}-x^{\lambda_{j}}\right\|_{\infty}<\frac{\epsilon}{2 \mu\left|d_{j}\right|} \quad \forall n \geq n_{j}, j=1,2, \ldots, \mu .
$$

Using (4.4) in (4.3), we get

$$
\left\|Q-Q_{n}^{\alpha}\right\|_{\infty}<\frac{\epsilon}{2} \quad \forall n \geq \max \left\{n_{j}, j=1,2, \ldots, \mu\right\} .
$$

Finally, combining (4.2) and (4.5) via the triangular inequality

$$
\left\|f-Q_{n}^{\alpha}\right\|_{\infty} \leq\|f-Q\|_{\infty}+\left\|Q-Q_{n}^{\alpha}\right\|_{\infty} \quad \forall n \geq \max \left\{n_{j}, j=1,2, \ldots, \mu\right\},
$$

we get $\left\|f-Q_{n}^{\alpha}\right\|_{\infty}<\epsilon$ for all $n \geq \max \left\{n_{j}, j=1,2, \ldots, \mu\right\}$. Hence, there exists a sequence $\left\{Q_{n}^{\alpha}\right\}_{n=1}^{\infty}$ of Bernstein $\alpha$-Müntz polynomials that converges uniformly to $f \in \mathcal{C}[0,1]$ and hence $\bigcup_{n=1}^{\infty} \operatorname{Span}\left\{1,\left(x^{\lambda_{1}}\right)_{n}^{\alpha},\left(x^{\lambda_{2}}\right)_{n}^{\alpha}, \ldots,\right\}$ is dense in $\mathcal{C}[0,1]$ with respect to the supremum norm.

The next Theorem 4.2 and Theorem 4.3 can be proved with arguments similar to those of Theorem 4.1.

THEOREM 4.2 (Fractal version of Müntz' second theorem in $\mathcal{C}[0,1]$ ). Let the partition $\Delta=\left\{x_{1}, x_{2}, \ldots, x_{N}\right\}$ of $I=[0,1]$ satisfy $0=x_{1}<x_{2}<\cdots<x_{N}=1$, and let $\alpha=\left(\alpha_{1}, \alpha_{2}, \ldots, \alpha_{N-1}\right) \in(-1,1)^{N-1}$ be a scaling vector. Suppose that $\left\{\lambda_{j}\right\}_{j=1}^{\infty}$ is a sequence of distinct real numbers satisfying $1 \leq \lambda_{j} \rightarrow \infty$. Then, for every scaling vector $\alpha$, the set $\bigcup_{n=1}^{\infty} \operatorname{Span}\left\{1,\left(x^{\lambda_{1}}\right)_{n}^{\alpha},\left(x^{\lambda_{2}}\right)_{n}^{\alpha}, \ldots\right\}$ is dense in $\mathcal{C}[0,1]$ with respect to the supremum norm if $\sum_{j=1}^{\infty} \lambda_{j}^{-1}=\infty$. 
THEOREM 4.3. Let the partition $\Delta=\left\{x_{1}, x_{2}, \ldots, x_{N}\right\}$ of $I=[0,1]$ satisfy $0=x_{1}<x_{2}<\cdots<x_{N}=1$, and let $\alpha=\left(\alpha_{1}, \alpha_{2}, \ldots, \alpha_{N-1}\right) \in(-1,1)^{N-1}$ be a scaling vector. If $\operatorname{Span}\left\{f_{j}: j \in \mathbb{N}\right\}$ is dense in $\mathcal{C}[0,1]$, then, for every scaling vector $\alpha$, the set $\bigcup_{n=1}^{\infty} \operatorname{Span}\left\{f_{j, n}^{\alpha}: j \in \mathbb{N}\right\}$ also is.

THEOREM 4.4 (Fractal version of the full Müntz theorem in $L^{p}[0,1], 1 \leq p<\infty$ ). Let the partition $\Delta=\left\{x_{1}, x_{2}, \ldots, x_{N}\right\}$ of $I=[0,1]$ satisfy $0=x_{1}<x_{2}<\ldots,<x_{N}=1$, and let $\alpha=\left(\alpha_{1}, \alpha_{2}, \ldots, \alpha_{N-1}\right) \in(-1,1)^{N-1}$ be a scaling vector. Suppose that $\left\{\lambda_{j}\right\}_{j=1}^{\infty}$ is a sequence of distinct real numbers greater than $-1 / p$. Then, for every scaling vector $\alpha$, the set $\bigcup_{n=1}^{\infty} \operatorname{Span}\left\{\hat{\mathcal{F}}_{n}^{\alpha}\left(x^{\lambda_{1}}\right), \hat{\mathcal{F}}_{n}^{\alpha}\left(x^{\lambda_{2}}\right), \ldots\right\}$ is dense in $L^{p}[0,1]$ with respect to the $L^{p}$-norm if $\sum_{j=1}^{\infty}\left(\lambda_{j}+1\right) /\left(\left(\lambda_{j}+\frac{1}{p}\right)^{2}+1\right)=\infty$.

Proof. Let $f \in L^{p}[0,1]$, and let $\epsilon>0$ be given. From the classical full Müntz theorem [2] in $L^{p}[0,1]$, we obtain that $\operatorname{Span}\left\{x^{\lambda_{1}}, x^{\lambda_{2}}, \ldots,\right\}$ is dense in $L^{p}[0,1]$ if

$$
\sum_{j=1}^{\infty} \frac{\lambda_{j}+1}{\left(\lambda_{j}+\frac{1}{p}\right)^{2}+1}=\infty
$$

where $\left\{\lambda_{j}\right\}_{j=1}^{\infty}$ is a sequence of distinct real numbers greater than $\frac{-1}{p}$. Hence, there exists a Müntz polynomial $Q \in \operatorname{Span}\left\{x^{\lambda_{1}}, x^{\lambda_{2}}, \ldots,\right\}$ such that

$$
\|f-Q\|_{\infty}<\frac{\epsilon}{2}
$$

Next, using (4.1), we get $\hat{\mathcal{F}}_{n}^{\alpha}(Q)=Q_{n}^{\alpha}$ for all $n \in \mathbb{N}$.

Following the same steps as in the proof of Theorem 4.1 yields

$$
\left\|Q-Q_{n}^{\alpha}\right\|_{\infty}<\frac{\epsilon}{2}
$$

Finally, combining (4.6) with (4.7) via the triangular inequality gives

$$
\left\|f-Q_{n}^{\alpha}\right\|_{\infty} \leq\|f-Q\|_{\infty}+\left\|Q-Q_{n}^{\alpha}\right\|_{\infty}
$$

and hence we get $\left\|f-Q_{n}^{\alpha}\right\|_{\infty}<\epsilon$. That is, there exists a double sequence $\left\{Q_{n}^{\alpha}\right\}_{n=1}^{\infty}$ of Bernstein $\alpha$-Müntz polynomials which converges to $f \in L^{p}[0,1]$, and hence the space $\bigcup_{n=1}^{\infty} \operatorname{Span}\left\{\hat{\mathcal{F}}_{n}^{\alpha}\left(x^{\lambda_{1}}\right), \hat{\mathcal{F}}_{n}^{\alpha}\left(x^{\lambda_{2}}\right), \ldots,\right\}$ is dense in $L^{p}[0,1]$.

The next theorem can be proved with arguments similar to those of the previous theorem.

THEOREM 4.5 (Fractal version of Müntz' first theorem in $L^{p}[0,1]$ ). Let the partition $\Delta=\left\{x_{1}, x_{2}, \ldots, x_{N}\right\}$ of $I=[0,1]$ satisfy $0=x_{1}<x_{2}<\cdots<x_{N}=1$, and let $\alpha=\left(\alpha_{1}, \alpha_{2}, \ldots, \alpha_{N-1}\right) \in(-1,1)^{N-1}$ be a scaling vector. Suppose that $\left\{\lambda_{j}\right\}_{j=1}^{\infty}$ is a sequence of distinct and positive real numbers satisfying $-1 / 2<\lambda_{j} \rightarrow \infty$. Then, for every scaling vector $\alpha$, the set $\bigcup_{n=1}^{\infty} \operatorname{Span}\left\{\hat{\mathcal{F}}_{n}^{\alpha}\left(x^{\lambda_{1}}\right), \hat{\mathcal{F}}_{n}^{\alpha}\left(x^{\lambda_{2}}\right), \ldots\right\}$ is dense in $L^{p}[0,1]$ with respect to the supremum norm if $\sum_{j=1}^{\infty} \lambda_{j}^{-1}=\infty$.

In the next theorem, we investigate the constrained approximation problem of Müntz polynomials using the result of [30].

THEOREM 4.6. Let $\Phi$ and $\Psi$ be continuous Müntz polynomials on $[0,1]$ such that $\Phi(x)>\Psi(x)$ for all $x \in[0,1]$. Let $\Delta=\left\{x_{1}, x_{2}, \ldots, x_{N}\right\}$ be a partition of $[0,1]$ satisfying $0=x_{1}<x_{2}<\cdots<x_{N}=1$. For $n \in \mathbb{N}$, let $\Phi_{n}^{\alpha}$ be the Bernstein $\alpha$-fractal function of $\Phi$ corresponding to the IFS $\mathcal{I}_{n}$. For each $n \in \mathbb{N}, \Phi_{n}^{\alpha}(x) \geq \Psi(x)$ for all $x \in[0,1]$, and the sequence $\left\{\Phi_{n}^{\alpha}\right\}_{n=1}^{\infty}$ of Bernstein $\alpha$-Müntz polynomials converges uniformly to $\Phi$ if the scaling factors are such that

$$
0 \leq \alpha_{i}<\min \left\{\frac{m(\Phi-\Psi, i)}{M_{n}(\Phi)-m(\Psi)}, 1\right\}, \quad i \in \mathbb{N}_{N-1}
$$


where $M_{n}(\Phi)=\max _{x \in I} B_{n}(\Phi, x), m(\Phi-\Psi, i)=\min _{x \in I}(\Phi-\Psi)\left(L_{i}(x)\right)$, and $m(\Psi)=\min _{x \in I} \Psi(x)$.

Proof. Since $\Phi(x) \geq \Psi(x)$ for all $x \in[0,1]$ and $B_{n}(\Phi, x)$ interpolates $\Phi$ at $x_{1}$ and $x_{N}$, it is easy to verify that $M_{n}(\Phi)-m(\Psi)>0$ and $m(\Phi-\Psi, i)>0$ for all $i \in \mathbb{N}_{N-1}$. From (3.2), we notice that the Bernstein $\alpha$-fractal function $\Phi_{n}^{\alpha}$ obeys the following functional equation:

$$
\Phi_{n}^{\alpha}\left(L_{i}(x)\right)=\alpha_{i} \Phi_{n}^{\alpha}(x)+\Phi\left(L_{i}(x)\right)-\alpha_{i} B_{n}(\Phi, x), \quad x \in I, i \in \mathbb{N}_{N-1} .
$$

For each $n \in \mathbb{N}$ this yields the values of $\Phi_{n}^{\alpha}$ at $(N-1)^{p+2}+1$ distinct points in $I$ at the $(p+1)$ st iteration by using the values of $\Phi_{n}^{\alpha}$ at $(N-1)^{p+1}+1$ distinct points in $I$ computed at the $p$-th iteration. Since $\Phi(x)>\Psi(x)$ for all $x \in I$, it is straightforward to see that $\Phi_{n}^{\alpha}\left(x_{i}\right)=\Phi\left(x_{i}\right)>\Psi\left(x_{i}\right)$ for $i \in \mathbb{N}_{N-1}$. Therefore, to prove $\Phi_{n}^{\alpha}(z)>\Psi(z)$ for all $z \in I$, it is enough to show that $\Phi_{n}^{\alpha}(x)>\Psi(x)$ for $x \in I$ implies that $\Phi_{n}^{\alpha}\left(L_{i}(x)\right)>\Psi\left(L_{i}(x)\right)$ for $x \in I$ and for all $i \in \mathbb{N}_{N-1}$. Assume that $\Phi_{n}^{\alpha}(x)>\Psi(x)$ for $x \in I$. We have to show that

$$
\alpha_{i} \Phi_{n}^{\alpha}(x)+\Phi\left(L_{i}(x)\right)-\alpha_{i} B_{n}(\Phi, x)-\Psi\left(L_{i}(x)\right)>0, \quad x \in I .
$$

Since $\alpha_{i} \geq 0, i \in \mathbb{N}_{N-1}$, from the definitions of $M_{n}(\Phi)$ and $m(\Psi)$, it is easy to show that

$$
\alpha_{i} \Phi_{n}^{\alpha}(x)+\Phi\left(L_{i}(x)\right)-\alpha_{i} B_{n}(\Phi, x)-\Psi\left(L_{i}(x)\right) \geq \alpha_{i}\left(m(\Psi)-M_{n}(\Phi)\right)+m(\Phi-\Psi, i) .
$$

From (4.8), we can then infer that $\alpha_{i}<\frac{m(\Phi-\Psi, i)}{M_{n}(\Phi)-m(\Psi)}$ ensures (4.8) and thus the conclusion. $\square$

5. Bernstein $\alpha$-fractal Chebyshev series. In this section, we establish the fractal analogue of the Chebyshev series.

We consider $\mathcal{C}[-1,1]$ equipped with the inner product

$$
\langle f, g\rangle=\frac{2}{\pi} \int_{-1}^{1} f(x) g(x) \frac{1}{\sqrt{1-x^{2}}} d x .
$$

The Chebyshev system $\left\{\frac{T_{0}(x)}{\sqrt{2}}, T_{1}(x), T_{2}(x), \ldots\right\}$, where

$$
T_{0}(x)=1, \quad T_{1}(x)=x, \quad T_{n+1}(x)=2 x T_{n}(x)-T_{n-1}(x), \quad n \geq 2,
$$

is orthonormal with respect to the aforementioned inner product. The Chebyshev series of $f \in \mathcal{C}[-1,1]$ is given by

$$
f(x) \sim \sum_{j=0}^{\infty} d_{j} T_{j}(x), \quad \text { where } \quad d_{j}=\frac{2}{\pi} \int_{-1}^{1} f(x) T_{j}(x) \frac{1}{\sqrt{1-x^{2}}} d x .
$$

Let us define the Chebyshev sum of order $m$ as

$$
S_{m}(x)=\sum_{j=0}^{m} d_{j} T_{j}(x), \quad \forall x \in[-1,1] .
$$

Now, we define the operator $U_{m}: \mathcal{C}[-1,1] \rightarrow \operatorname{Span}\left\{\frac{T_{0}(x)}{\sqrt{2}}, T_{1}(x), T_{2}(x), \ldots\right\}$ as

$$
U_{m}(f)(x)=S_{m}(x), \quad \forall x \in[-1,1] .
$$

We obtain from [13] that

$$
\left\|U_{m} f-f\right\|_{\infty} \leq \frac{C K \log m}{m} .
$$


Let $\Delta=\left\{x_{1}, x_{2}, \ldots, x_{N}\right\}$ be a partition of $[-1,1]$ with $-1=x_{1}<x_{2}<\cdots<x_{N}=1$. Let $U_{m, n}^{\alpha}=\mathcal{F}_{n}^{\alpha} \circ U_{m}$ be an operator such that $U_{m, n}^{\alpha}(f)=\mathcal{F}_{n}^{\alpha}\left(U_{m}(f)\right)=\left(U_{m}(f)\right)_{n}^{\alpha}$. Now,

$$
\left\|U_{m, n}^{\alpha}(f)-U_{m}(f)\right\|_{\infty} \leq \sum_{j=0}^{m}\left|d_{j}\right|\left\|\mathcal{F}_{n}^{\alpha}\left(T_{j}\right)-T_{j}\right\|_{\infty} .
$$

Using the definition of $\mathcal{F}_{n}^{\alpha}\left(T_{j}\right)$ in the previous equation, we obtain

$$
\left\|U_{m, n}^{\alpha}(f)-U_{m}(f)\right\|_{\infty} \leq \sum_{j=0}^{m}\left|d_{j}\right|\left\|\left(T_{j}\right)_{n}^{\alpha}-T_{j}\right\|_{\infty} .
$$

Using Theorem 3.1, one can observe that the sequence $\left\{\left(T_{j}\right)_{n}^{\alpha}\right\}_{n=1}^{\infty}$ converges uniformly to $T_{j}$ for each $j=1,2, \ldots, m$. Hence, for given $\epsilon>0$, there exists $n_{j} \in \mathbb{N}$ such that

$$
\left\|\left(T_{j}\right)_{n}^{\alpha}-T_{j}\right\|_{\infty}<\frac{\epsilon}{2(m+1)\left|d_{j}\right|}, \quad \forall n \geq n_{j}, j=0,1,2, \ldots, m .
$$

Using (5.3) in (5.2), we get

$$
\left\|U_{m, n}^{\alpha}(f)-U_{m}(f)\right\|_{\infty}<\frac{\epsilon}{2} \quad \forall n \geq \max \left\{n_{j}: j=0,1,2, \ldots, m\right\} .
$$

Finally, using (5.1) and (5.4) in the triangular inequality

$$
\left\|U_{m, n}^{\alpha}(f)-f\right\|_{\infty} \leq\left\|U_{m, n}^{\alpha}(f)-U_{m} f\right\|_{\infty}+\left\|U_{m} f-f\right\|_{\infty}
$$

we get $\left\|U_{m, n}^{\alpha}(f)-f\right\|_{\infty}<\epsilon+\frac{C K \log m}{m}$. This proves the following theorem.

THEOREM 5.1. Let $f \in \mathcal{C}[-1,1]$. Then for every scaling vector $\alpha$, the fractal Chebyshev series of $f$ converges uniformly to $f$ as $m \rightarrow \infty$ and $n \rightarrow \infty$, where $m, n \in \mathbb{N}$.

6. Conclusion. In this paper, using Bernstein polynomials and the theory of $\alpha$-fractal functions as major components, we have presented the Bernstein $\alpha$-fractal functions as a tool to approximate univariate continuous functions defined on a real compact interval. In our approach, the convergence of Bernstein $\alpha$-fractal functions does not require any condition on the scaling factors. For the sequence $\left\{f_{n}(x)\right\}_{n=1}^{\infty}$ of continuous functions that converges uniformly to a continuous function $f$, we have identified the double sequence $\left\{\left\{\mathcal{I}_{n, l}^{\dagger}\right\}_{l=1}^{\infty}\right\}_{n=1}^{\infty}$ of IFSs so that the corresponding double sequence $\left\{\left\{f_{n, l}^{\alpha}(x)\right\}_{l=1}^{\infty}\right\}_{n=1}^{\infty}$ of $\alpha$-fractal functions converges to $f$. We have also studied the approximation properties of Bernstein $\alpha$-fractal functions in the space of continuous functions and $L^{p}$-spaces. The results are based on the analysis of the norm estimates for the associated $\alpha$-operator $\mathcal{F}_{n}^{\alpha}$, which maps a function $f$ to its $n$-th Bernstein $\alpha$-fractal function $f_{n}^{\alpha}$. These estimates are applied to the particular case of Müntz polynomials to obtain the fractal analogue of (i) the full Müntz theorems and (ii) Müntz first and second theorems in the space of continuous functions and $L^{p}$ spaces. Without imposing any condition on the scaling factors, we proved that $\bigcup_{n=1}^{\infty} \operatorname{Span}\left\{f_{j, n}^{\alpha}: j \in \mathbb{N}\right\}$ is dense in the space of continuous functions and in $L^{p}$ if $\operatorname{Span}\left\{f_{j}: j \in \mathbb{N}\right\}$ is dense in the above function spaces. We have solved the constrained approximation problem of Müntz polynomials. Finally, we have developed the Bernstein $\alpha$-fractal Chebyshev series.

Acknowledgments. The author is grateful to the referees for extensive comments and constructive criticisms. The valuable comments and suggestions led to several improvements in the paper.

\section{REFERENCES}

[1] M. F. BARNSLEy, Fractal functions and interpolation, Constr. Approx., 2 (1986), pp. 303-329. 
[2] P. Borwein And T. ERdéLyi, The full Müntz theorem in $\mathcal{C}[0,1]$ and $L_{1}[0,1]$, J. London Math. Soc., 54 (1996), pp. 102-110.

[3] A. K. B. ChAND AND G. P. KAPOOR, Generalized cubic spline fractal interpolation functions, SIAM J. Numer. Anal., 44 (2006), pp. 655-676.

[4] - Hidden variable bivariate fractal interpolation surfaces, Fractals, 11 (2003), pp. 277-288.

[5] A. K. B. ChAND AND N. ViJEnder, Positive blending Hermite rational cubic spline fractal interpolation surfaces, Calcolo, 52 (2015), pp. 1-24.

[6] - A new class of fractal interpolation surfaces based on functional values, Fractals, 24 (2016), Art. No. 1650007, 17 pages.

[7] A. K. B. Chand, N. Vijender, And R. P. Agarwal, Rational iterated function system for positive/monotonic shape preservation, Adv. Difference Equ, 2014, Art. No. 30, 19 pages.

[8] A. K. B. CHAND, N. VIJENDER, M. A. NAVASCUÉS, Shape preservation of scientific data through rational fractal splines, Calcolo, 51 (2013), pp. 329-362.

[9] - Convexity/concavity and stability aspects of rational cubic fractal interpolation surfaces, Comput. Math. Model, 28 (2017), pp. 407-430.

[10] E. W. ChEnEY, Introduction to Approximation Theory, McGraw-Hill, New York, 1996

[11] S. G. GAL, Shape Preserving Approximation by Real and Complex Polynomials, Birkhäuser, Boston, 2008.

[12] J. S. HwANG, A note on Bernstein and Müntz-Szász theorems with applications to the order statistics, Ann. Inst. Statist. Math., 30 (1978), pp. 167-176.

[13] Y.-S. LIANG AND Q. ZHANG, A type of fractal interpolation functions and their fractional calculus, Fractals, 24 (2016), Art. No. 1650026, 9 pages.

[14] C. MÜNTZ, Über den Approximationsatz von Weierstra $\beta$, in Mathematische Abhandlungen Hermann Amandus Schwarz, C. Caratheodory, G. Hessenberg, E. Landau, and L. Lichtenstein, eds., Springer, Berlin, 1914, pp. 303-312.

[15] Md. Nasim Akhtar, M. Guru Prem Prasad, and M. A. Navascués, Box dimensions of $\alpha$-fractal functions, Fractals, 24 (2016), Art. No. 1650037, 13 pages.

[16] M. A. NAVASCUÉs, Fractal trigonometric approximation, Electron. Trans. Numer. Anal., 20 (2005), pp. 64-74. http://etna.ricam.oeaw.ac.at/vol.20.2005/pp64-74.dir/pp64-74.pdf

[17] - Fractal polynomial interpolation, Z. Anal. Anwendungen, 24 (2005), pp. 401-418.

[18] — Non-smooth polynomials, Int. J. Math. Anal. (Ruse), 1 (2007), pp. 159-174.

[19] - Fractal approximation, Complex Anal. Oper. Theory, 4 (2010), pp. 953-974.

[20] - Fractal Haar system, Nonlinear Anal., 74 (2011), pp. 4152-4165.

[21] - Fractal bases of $L_{p}$ spaces, Fractals, 20 (2012), pp. 141-148.

[22] M. A. NAVAScuÉs AND A. K. B. ChAnd, Fundamental sets of fractal functions, Acta Appl. Math., 100 (2008), pp. 247-261.

[23] M. A. NAVASCUÉS AND M. V. SEBAStiáN, Generalization of Hermite functions by fractal interpolation, J. Approx. Theory., 131 (2004), pp. 19-29.

[24] — Smooth fractal interpolation, J. Inequal. Appl., 2006, Art. No. 78734, 20 pages.

[25] H. K. RYU, Choice of representation system for economic analysis, Appl. Econ. Lett., 10 (2003), pp. 863-866.

[26] L. SchwartZ, Étude des Sommes d'Exponentielles, Harmann, Paris, 1959.

[27] A. R. Siegel, On the Müntz-Szász theorem for $\mathcal{C}[0,1]$, Proc. Amer. Math. Soc., 36 (1972), pp. 161-166.

[28] O. SZÁsz, Über die Approximation stetiger Funktionen durch lineare Aggregate von Potenzen, Math. Ann., 77 (1916), pp. 482-496.

[29] P. Viswanathan AND A. K. B. Chand, $\alpha$-fractal rational splines for constrained interpolation, Electron. Trans. Numer. Anal., 41 (2014), pp. 420-442.

http://etna.ricam.oeaw.ac.at/vol.41.2014/pp420-442.dir/pp420-442.pdf

[30] P. Viswanathan, M. A. NAVASCuÉs, AND A. K. B. Chand, Fractal perturbation preserving fundamental shapes: bounds on the scale factors, J. Math. Anal. Appl., 419 (2014), pp. 804-817.

[31] _ Associate fractal functions in $L^{p}$-spaces and in one-sided uniform approximation, J. Math. Anal. Appl., 433 (2016), pp. 862-876. 